\title{
In Vitro Approaches to Environmental Pollutants: New Models, Endpoints, and Strategies
}

\author{
doi:10.14573/altex.1901181
}

Environmental pollution is a relevant source of contaminants affecting human health and ecosystems. Generally, target organisms are chronically exposed to a multi-constituent mixture in which each pollutant is present at a low concentration. In the last decades, the use of in vitro methods has increased in environmental toxicology and ecotoxicology due to technological development and the availability of validated assays and protocols. The use of experimental models, endpoints, and in vitro approaches in environmental toxicology was discussed at the meeting "Environmental contaminants and in vitro methodologies" held in Genova in May 2018, hosted by Italy's National Research Council, Research Area of Genova, and organized by CELLTOX Italian Association of in vitro Toxicology. The meeting sessions were chaired by Diego Baderna, President of CELLTOX, and Susanna Alloisio.

Diego Baderna, Istituto di Ricerche Farmacologiche Mario Negri IRCCS, focused on air quality, presenting the strategy adopted by the LIFE+ COBRA project to investigate the toxicity of brake-derived particulate matter on in vitro human bronchial cells and ecological plant targets. Brake wear can represent more than $10 \%$ of the total particles emitted by road traffic. The studies were done on particulate matter generated under laboratory conditions using test benches that simulate vehicle braking cycles of traditional or innovative brake pads. PM2.5 particles were sampled using impactor devices equipped with Teflon filters and sonicated to detach particles from the filter. Effects on cell proliferation, cytotoxicity, and sublethal responses (oxidative stress, DNA damage, and mitochondrial membrane potential) were measured in exposed human immortalized bronchial cells BEAS-2B cells. Non-airborne debris was collected on a stainless-steel tray placed on the test bench and its ecotoxicological impact on soil plants, freshwater crustaceans, and microalgae was evaluated. Particles derived from cementitious pads were less toxic in vitro than those from traditional pads with regard to cell proliferation and sublethal effects; a similar trend was found also in plants exposed to debris-contaminated soils (Maiorana et al., 2019).

Chiara Gambardella, IAS CNR Genova, presented a new approach to ecotoxicity assessment of emerging contaminants in the marine environment based on behavioral responses of invertebrates. Traditional ecotoxicological endpoints are not always sufficiently sensitive, therefore there is an urgent need to develop alternative endpoints. Behavioral responses are promising indicators of environmental stress, since they provide an indication of toxicity before organism death (Melvin and Wilson, 2013). The swimming behavior of crustacean larvae (Amphibalanus amphitrite, Artemia franciscana), rotífers (Brachionus plicatilis), echinoderm larvae (Paracentrotus lividus), and the frequency of pulsations of the juvenile stage (ephyra) of cnidarians (Aure- lia $s p$.) have proven to successfully detect toxicity of traditional and emerging contaminants, such as pharmaceuticals, nano-, and micromaterials (Morgana et al., 2016; Estevez-Calvar et al., 2017; Faimali et al., 2017; Gambardella et al., 2017).

Valentina Giussani, ARPAL and DISTAV-University of Genova, proposed an approach for environmental risk assessment of harmful microalgae. Some microalgal species are known to negatively affect ecosystems, other organisms, and/or human activities by production of toxins. Occurrence of these species, which is often associated with harmful algal blooms (HABs), seems to have increased worldwide over recent decades but no standard protocols to assess the associated environmental risk exist. Ostreopsis cf. ovata is one of the most common benthic harmful dinoflagellates along Italian coasts. Several algal samples collected during different phases of its growth were tested on Artemia salina and other model organisms from different taxa (other crustaceans, larvae and juveniles of sea urchins and polyps and ephyrae of jellyfish) to understand how toxic effects vary with direct/ indirect exposures and life cycle (Faimali et al., 2012; Privitera et al., 2012; Giussani et al., 2016). Nauplii of $A$. salina and ephyrae of the jellyfish Aurelia sp. were the most sensitive to direct contact with $O$. ovata culture. The study demonstrates an approach that could be applied as a standard protocol to study the ecotoxicological impact of harmful algal species.

Francesca Caloni, Università degli Studi di Milano-DIMEVET, presented a lecture entitled "Emerging contaminants of natural and synthetic origin: in vitro approach." Possible adverse effects of contaminants of emerging concern (CECs), be they natural or synthetic, alone or in a mixture, are of interest for risk assessment. Research is aiming on the one hand at the protection of animals used for scientific purposes (Directive 2010/63 EU) by application of the 3Rs (Reduce, Refine, Replace), and on the other hand at a predictive interdisciplinary science for risks related to the environment, humans, and animals. The presentation focused on the evaluation of the effects of CECs including natural compounds such as beauvericin, an emerging mycotoxin, and ciguatoxin (Coccini et al., 2017), an emerging marine toxin, as well as synthetic substances like the herbicide glyphosate, as a chemical or in a formulation (Perego et al., 2017). Different cell-based assays for the evaluation of neurotoxicity, endocrine disruptor effects, but also bioavailability, mechanism of action, mode of action, metabolism and transport were applied. The importance of in vitro approaches as part of an integrated testing strategy (ITS) (Rovida et al., 2015), and species-specific models, including organoids, was discussed.

Isabella De Angelis, Istituto Superiore di Sanità spoke on in vitro approaches for nanotoxicology. Nanomaterials (NM) are 
characterized by peculiar properties derived from a high surface area to volume ratio. NM can present risks to the health of operators, consumers, and the environment. In vitro assays are relevant in the early stages of hazard identification of NMs and may contribute to the evaluation of in vivo results and for NM categorization. Cytotoxicity, apoptosis, oxidative stress, genotoxicity, and inflammatory processes are the parameters most frequently investigated in vitro. To date, there are no standardized in vitro tests or specific experimental protocols for the toxicological evaluation of NMs. Essential requirements for producing robust and significant in vitro data include accurate characterization of the NM, including definition of the chemical-physical properties, before, during, and after the test procedure; use of a realistic dosimetry system related to the possible exposure scenarios and of a reasonable range of doses (at least 4-5 different concentrations); use of multiple cell lines (preferably of human origin) representative of possible target organs; and presence of internal controls (positive and negative) and of reference $\mathrm{NM}(\mathrm{s})$ for intra and inter-laboratory comparison.

Laura Canesi, DISTAV-University of Genoa, gave a presentation entitled "Alternative methods for the screening of emerging contaminants", focusing first on the use of unconventional model organisms for studying marine pollutants (Fabbri et al., 2014) and then on in vitro animal and human models to evaluate the adverse effects of endocrine disruptors and obesogens (Balbi et al., 2017; Grasselli et al., 2017).

Speakers and audience participated in a round table discussion on the integration of different disciplines to challenge old and emerging problems in environmental toxicology. Sharing knowledge, through the presentation of technologies applied in different areas of research, may be a starting point for the creation of an interactive platform, with a toxicological vision involving ecosystems, animals, and humans in relation to health and safety. Meetings like this one, especially addressed to students and young researchers, and involving local environmental protection agencies, universities, and research centers, can contribute to forming a new community of experts working for a global science and can promote cooperation among different research fields and institutions.

\section{References}

Balbi, T., Ciacci, C., Grasselli, E. et al. (2017). Utilization of Mytilus digestive gland cells for the in vitro screening of potential metabolic disruptors in aquatic invertebrates. Comp Biochem Physiol C Toxicol Pharmacol 191, 26-35. doi:10.1016/j. cbpc.2016.08.009

Coccini, T., Caloni, F. and De Simone, U. (2017). Human neuronal cell based assay: A new in vitro model for toxicity evaluation of ciguatoxin. Environ Toxicol Pharmacol 52, 200-213. doi:10.1016/j.etap.2017.04.003

Estevez-Calvar, N., Canesi, L., Montagna, M. et al. (2017). Adverse effects of the SSRI antidepressant sertraline on early life stages of marine invertebrates. Mar Environ Res 128, 88-97. doi:10.1016/j.marenvres.2016.05.021

Fabbri, R., Montagna, M., Balbi, T. et al. (2014). Adaptation of the bivalve embryotoxicity assay for the high throughput screening of emerging contaminants in Mytilus galloprovincialis. Mar Environ Res 99, 1-8. doi:10.1016/j.marenvres.2014.05.007

Faimali, M., Giussani, V., Piazza, V. et al. (2012). Toxic effects of harmful benthic dinoflagellate Ostreopsis ovata on invertebrate and vertebrate marine organisms. Mar Environ Res 76, 97-107. doi:10.1016/j.marenvres.2011.09.010

Faimali, M., Gambardella, C., Costa, E. et al. (2017). Old model organisms and new behavioral end-points: Swimming alteration as an ecotoxicological response. Mar Environ Res 128, 36-45. doi:10.1016/j.marenvres.2016.05.006

Gambardella, C., Morgana, S., Ferrando, S. et al. (2017). Effects of polystyrene microbeads in marine planktonic crustacean. Ecotox Environ Saf 145, 250-257. doi:10.1016/j.ecoenv.2017.07.036

Giussani, V., Costa, E., Pecorino, D. et al. (2016). Effects of the harmful dinoflagellate Ostreopsis cf. ovata on different life cycle stages of the common moon jellyfish Aurelia sp. Harmful Algae 57, 49-58. doi:10.1016/j.hal.2016.05.005

Grasselli, E., Canesi, L., Portincasa, P. et al. (2017). Models of non-alcoholic fatty liver disease and potential translational value: The effects of 3,5-L-diiodothyronine. Ann Hepatol 16, 707-719. doi:10.5604/01.3001.0010.2713

Maiorana, S., Teoldi, F., Silvani, S. et al. (2019). Phytotoxicity of wear debris from traditional and innovative brake pads. Environ Int 123, 156-163. doi:10.1016/j.envint.2018.11.057

Melvin, S. D. and Wilson, S. P. (2013). The utility of behavioural studies for aquatic toxicology testing: A meta-analysis. Chemosphere 93, 2217-2223. doi:10.1016/j.chemosphere.2013.07.036

Morgana, S., Gambardella, C., Falugi, C. et al. (2016). Swimming speed alteration in the early developmental stages of Paracentrotus lividus sea urchin as ecotoxicological endpoint. Mar Environ Res 115, 11-19. doi:10.1016/j.marenvres.2016.01.007

Perego, M. C., Schutz, L. F., Caloni, F. et al. (2017). Evidence for direct effects of glyphosate on ovarian function: Glyphosate influences steroidogenesis and proliferation of bovine granulosa but not theca cells in vitro. J Appl Toxicol 37, 692-698. doi:10.1002/jat.3417

Privitera, D., Giussani, V., Isola, G. et al. (2012). Toxic effects of Ostreopsis ovata on larvae and juveniles of Paracentrotus lividus. Harmful Algae 18, 16-23. doi:10.1016/j.hal.2012.03.009

Rovida, C., Alépée, N., Api, A. M. et al. (2015). Integrated testing strategies (ITS) for safety assessment. ALTEX 32, 25-40. doi:10.14573/altex.1411011

Susanna Alloisio' ${ }^{1}$, Chiara Gambardella ${ }^{2}$, Valentina Giussani ${ }^{3}$, Marco Faimali ${ }^{2}$, Nathalie Steimberg ${ }^{4}$, Isabella De Angelis ${ }^{5}$, Francesca Caloni ${ }^{6}$ and Diego Baderna ${ }^{7}$

${ }^{1}$ ETT Spa, Genoa, Italy; ${ }^{2}$ The Institute for the study of Anthropic Impacts and Sustainability in the marine environment (IAS), CNR, Genoa, Italy; ${ }^{3}$ Dipartimento di Scienze della Terra, dell'Ambiente e della Vita (DISTAV), University of Genoa, Genoa, Italy; ${ }^{4}$ Department of Clinical and Experimental Sciences (DSCS), School of Medicine, University of Brescia, Brescia, Italy; ${ }^{5}$ Health and Environment Department, Istituto Superiore di Sanità, Roma, Italy; ${ }^{6}$ Department of Veterinary Medicine (DIMEVET), Università degli Studi di Milano, Milan, Italy; ${ }^{7}$ Department of Environmental Health Sciences, Istituto di Ricerche Farmacologiche Mario Negri IRCCS, Milan, Italy 